\title{
Corrigendum
}

\section{On the spectral radius of positive operators}

\author{
Y.A. Abramovich, C.D. Aliprantis, and O. Burkinshaw \\ Department of Mathematical Sciences, IUPUI, Indianapolis, IN 46202-3216, USA
}

Math. Z. 211, 593-607 (1992)

Professor J.J. Grobler has kindly pointed out to us that Theorems 4.4 and 5.3 in our paper [1] are false as stated and need correction. The error in the proofs is the tacit claim that $\widehat{T}$ is a nonzero positive operator. To avoid this situation, we need to add the minor extra assumption $T^{2} \neq 0$, which is automatically satisfied in all places where Theorems 4.4 or 5.3 are invoked. Therefore, all results in [1] on the positivity of the spectral radius are correct as stated.

We follow the terminology and notation of [1] and start by stating and proving the correct version of Theorem 4.4.

Theorem 4.4 If a compact quasi-nilpotent positive operator $T: E \rightarrow E$ satisfies $T^{2} \neq 0$, then it has a non-trivial hyperinvariant closed ideal $J$ with $T(J) \neq\{0\}$.

Proof. Let $T: E \rightarrow E$ be a quasi-nilpotent compact positive operator on a Banach lattice such that $T^{2} \neq 0$. Let $N=\{x \in E: T|x|=0\}$ be the null ideal of $T$ and define the operator $\hat{T}: E / N \rightarrow E / N$ by $\hat{T}[x]=[T x]$. Clearly $\hat{T}$ is a quasi-nilpotent compact opertor, and we also claim that $\hat{T}>0$. Indeed, if $x>0$ satisfies $T^{\frac{2}{2}} x>0$, then $T x \notin N$, and hence $\hat{T}[x]=[T x]>0$ in $E / N$, which shows that $\hat{T} \neq 0$.

Now, by $[1$, Theorem 4.3], there exists a non-trivial hyperinvariant closed ideal $\hat{J}$ for $\hat{T}$. Let $J=\{x \in E:[x] \in \hat{J}\}$. Since the quotient map $x \mapsto[x]$ is a lattice homomorphism, it follows that $J$ is a non-trivial closed ideal in $E$ invariant under $T$. We claim that $T(J) \neq\{0\}$. To see this pick some $0<x \in J$ satisfying $0<[x] \in \hat{J}$ and notice that $T x>0$. Otherwise, $T x=0$ implies $x \in N$, that is, $[x]=0$, which is a contradiction.

Finally, notice that $J$ is a hyperinvariant ideal for $T$. To verify this, let $S: E \rightarrow E$ be a positive operator that commutes with $T$. If $[x]=[y]$, i.e., if $x-y \in N$, then from $0 \leqq T|S x-S y| \leqq T S|x-y|=S T|x-y|=0$, we see that $S x-S y \in N$, that is, $[S x]=[S y]$. Therefore, $S$ defines an operator $\hat{S} \geqq 0$ on $E / N$ via the formula $\hat{S}[x]=[S x]$. As $\hat{S}$ commutes with $\widehat{T}$, the inclusion $\hat{S}(\hat{J}) \subseteq \hat{J}$ holds and consequently, $S(J) \subseteq J$.

The following simple example shows that in the absence of the condition $T^{2} \neq 0$ the second part of Theorem 4.4 need not be valid. Consider the positive operator $T: \mathbb{R}^{2} \rightarrow \mathbb{R}^{2}$ defined by $T(x, y)=(y, 0)$. It is easy to verify that $T^{2}=0$ and that the band $J=\{(x, 0): x \in \mathbb{R}\}$ is the only non-trivial hyperinvariant closed ideal for $T$. However, $T(J)=\{0\}$. The same example shows that the second 
part of Theorem 5.3 does not hold either. Here is the correct version of Theorem 5.3 .

Theorem 5.3 If $T: E \rightarrow E$ is an order continuous compact quasi-nilpotent positive operator, then there exists a non-trivial hyperinvariant band $B$ for $T$. Moreover, if $T^{2} \neq 0$, then the band $B$ can be chosen to satisfy $T(B) \neq\{0\}$.

Proof. If the null ideal $N=\{x \in E: T|x|=0\}$ is non-zero, then $N$ is a non-trivial $T$-hyperinvariant band. So, we can assume that $T$ is strictly positive. Let $F$ denote the closed ideal generated by the range of $T$ in $E$, i.e.,

$$
F=\{x \in E: \exists y \in E \text { such that }|x| \leqq T y\}^{-} .
$$

Clearly, $F$ is a hyperinvariant ideal for $T$. Since $T: F \rightarrow F$ is a strictly positive compact quasi-nilpotent operator, it follows from the above Theorem 4.4, that $T$, as an operator on $F$, has a non-trivial hyperinvariant closed ideal $J \subset F$. Since $F$ itself is $T$-hyperinvariant, it follows that $J$, considered as ideal in $E$, is also $T$-hyperinvariant. Now let $B$ the band generated by $J$ in $E$. By [1, Lemma 5.2], every positive operator commuting with $T$ is order continuous, and so $B$ is a $T$-hyperinvariant band. To finish the proof, we shall show that $B \neq E$. To see this, assume by way of contradiction that $B=E$. Hence, for each $x>0$ there exists a net $\left\{x_{\alpha}\right\}$ of $J$ such that $0 \leqq x_{\alpha} \uparrow x$. By the order continuity of $T$, we get $T x_{\alpha} \uparrow T x$, and by the compactness of $T$, we see that the increasing net $\left\{T x_{\alpha}\right\}$ is norm convergent in $E$. It follows that $\left\|T x_{\alpha}-T x\right\| \rightarrow 0$. Since $J$ is $T$ invariant, we get $\left\{T x_{x}\right\} \subseteq J$, and thus $T x \in J$ for all $x \in E$. The latter conclusion implies $J=F$, contrary to $J \neq F$.

To establish the second part, assume now that $T^{2} \neq 0$. In this case, we consider the operator $\hat{T}: E / N \rightarrow E / N$ defined by $\hat{T}[x]=[T x]$, where $N$ is again the null ideal of $T$. We claim that $\hat{T}>0$. Indeed, if we pick $x>0$ such that $T^{2} x>0$, then $T x \notin N$ and so $\hat{T}[x]>0$. Clearly, $\hat{T}$ is quasi-nilpotent and compact. Moreover, the arguments in the second paragraph of the proof of Theorem 5.3 in [1] show that $\hat{T}$ is order continuous. By the first case, there exists a non-trivial hyperinvariant band $\hat{D}$ of $E / N$. Let $D=\{x \in E:[x] \in \hat{D}\}$. The order continuity of the quotient map $x \mapsto[x]$ implies that $D$ is a non-trivial $T$-hyperinvariant band. To complete the proof it suffices to show that $T(D) \neq\{0\}$. To see this, pick any $0<x \in D$ satisfying $0<[x] \in \hat{D}$. We claim that $T x>0$. Otherwise, $T x=0$ would imply that $x \in N$, i.e., $[x]=0$, which is a contradiction.

We employed Theorem 4.4 in the proof of Theorem 4.9 in [1]. The reader should notice that the same proof works provided that we invoke the above version of Theorem 4.4 and observe that any power of the operator $C$ is non-zero.

Theorem 5.3 is used in the proof of Theorem 5.4 in [1]. The first part of the above version of Theorem 5.3 suffices for the proof of Theorem 5.4. The other place we use Theorem 5.3 is in the proof of Theorem 5.7. In this proof (which mimics that of Theorem 4.9) one must additionally notice that $E$ has the countable sup property. The latter follows from Lemma 3.11 in [1] by observing that $(L Q)^{3}$ is a strictly positive compact operator.

Acknowledgements. We thank Professor J.J. Grobler for pointing out our error and Professor A.W. Wickstead for several stimulating discussions on this matter.

\section{References}

1. Abramovich, Y.A., Aliprantis, C.D. and Burkinshaw, O.: On the spectral radius of positive operators. Math. Z. 211, 593-607 (1992) 